\title{
SITUACIÓN ECONÓMICA DE LOS MAYORES EN ESPAÑA: RENTA, CONSUMO Y AHORRO
}

\section{ECONOMIC SITUATION OF THE LARGEST IN SPAIN: INCOME, CONSUMPTION AND SAVING}

\author{
AUTORES \\ Ester Bódalo Lozano: Profesora en la Universidad de Murcia. (España) \\ ebodalo@um.es
}

\section{CURRÍCULUM VITAE}

Profesora en la Universidad de Murcia. Socióloga experta en análisis sociales de los mayores y discapacitados y de empleo de recursos económicos.

\section{RESUMEN}

Entre los muchos temas relacionados con la vejez, el relativo a la situación económica o al bienestar económico de nuestros mayores, está adquiriendo una gran relevancia, ya que condicionará las formas y estilos de vida de una cada vez más importante segmento de nuestra población, no solo desde el punto de vista cuantitativo (hoy en día representan sobre el 15,35\% del total de la población), sino también desde el nivel cualitativo; cada vez se llega a la vejez en mejores condiciones de todo tipo y presumiblemente con un mejor nivel adquisitivo, lo que hace mover un importante volumen de dinero en la economía española. 


\title{
PALABRAS CLAVE
}

Mayores - Renta - Situación económica - Ahorro - Consumo

\begin{abstract}
Among the many topics related to old age, on the economic situation or the economic welfare of our elderly, is becoming more relevant, since that will determine the ways and lifestyles of an increasingly important segment of our population, not only from a quantitative perspective (now represent about $15.35 \%$ of total population), but also from the qualitative level, each time you reach old age in better conditions of all types and presumably better purchasing power, which makes moving a significant amount of money in the Spanish economy.
\end{abstract}

\section{KEY WORDS}

Seniors - Income - Economic - Savings - Consumption

\section{ÍNDICE}

1. Introducción

2. Estudios sobre la situación económica de los mayores

3. Análisis de la situación económica

3.1. Evolución de los ingresos y gastos por edad.

4. La renta de las personas mayores

5. Consumo de los hogares cuyo sustentador principal tiene más de 65 años.

6. El ahorro para la vejez.

7. El consumo tras la jubilación

7.1. Las ayudas económicas familiares 
8. Bibliografía

\section{Introducción}

Entre los muchos temas relacionados con la vejez, el relativo a la situación económica o al bienestar económico de nuestros mayores, está adquiriendo una gran relevancia, ya que condicionará las formas y estilos de vida de una cada vez más importante segmento de nuestra población, no solo desde el punto de vista cuantitativo (hoy en día representan sobre el 15,35\% del total de la población), sino también desde el nivel cualitativo; cada vez se llega a la vejez en mejores condiciones de todo tipo y presumiblemente con un mejor nivel adquisitivo, lo que hace mover un importante volumen de dinero en la economía española.

Por lo que respecta a la situación económica de los mayores en España, debemos tener en cuenta que ésta ha mejorado sensiblemente, aunque por ello, no podemos dejar de hablar de bolsas de pobreza ligadas a la vejez. También es cierto, que algunos mitos sobre el mayor se han ido desvaneciendo, tales como su bajo poder adquisitivo, su mala salud o su conservadurismo extremo a la hora de probar nuevos productos y servicios (Grande Esteban, 1993). Por ello hay que estar atentos a los cambios que se están produciendo en los sistemas de valores y en las formas de afrontar la vida de este segmento de la población. Estos cambios van a suponer, - de hecho ya lo están suponiendo- una nueva disposición hacia el consumo y por tanto un nuevo estatuto de éste, dentro del hontanar del mayor (Sánchez Vera, 2000). 
Abordar el tema de la situación económica de los mayores en nuestro país no es sencillo, debido por un lado a la dificultad de encontrar datos desagregados de este grupo de edad, a la reticencia de la población ante las preguntas referidas a sus fuentes y niveles de ingresos y a la diversidad de sus necesidades, ya que cuando se trata de población anciana se dan los mayores contrastes en cuanto a experiencias vitales. Normalmente se habla de la tercera edad como grupo homogéneo, pero en la vejez no todo son rasgos comunes, sino que a veces la heterogeneidad y la diversidad es el rasgo dominante en este grupo de edad. A parte de la edad, la situación económica vendrá influida por una serie de variables tales como la situación económica patrimonial, el nivel de estudios, el estado de salud, el género, la situación familiar, la biografía laboral, etc.

\section{Estudios sobre la situación económica de los mayores}

En España, donde tan sólo en los últimos tiempos han comenzado a desarrollarse las bases de datos de origen fiscal, las Encuestas de Presupuestos Familiares del INE constituyen la fuente tradicionalmente utilizada para estudiar analizan la distribución de la renta, la pobreza o la posición económica de los diversos grupos de población. La principal ventaja de esta fuente frente a otras de tipo personal (como las estadísticas de salarios o pensiones), consiste en permitir el análisis del consumo y de la renta familiar disponible, en combinación con una amplia serie de características económicas y sociodemográficas de los individuos. Para el caso concreto del estudio de la población de mayor edad, la EPF permite un análisis integrado de los diversos tipos de renta de este colectivo en el marco del hogar, que resultaría imposible a partir de una combinación de estadísticas parciales. 
La amplia utilización de esta fuente no significa la inexistencia de problemas relacionados con el ámbito temporal, el diseño y la fiabilidad de la información en ella contenida. Por lo que respecta al primer aspecto, la escasa periodicidad de la encuesta, que viene publicándose a intervalos irregulares desde los años 60, constituye una limitación evidente. La existencia de encuestas comparables intermedias o posteriores permitiría un estudio más detallado de las tendencias (la Encuesta Continua de Presupuestos Familiares que viene realizándose desde 1.985 con carácter trimestral, posibilita un cierto seguimiento de los grandes agregados, pero el pequeño tamaño de su muestra no permite asegurar la bondad de los resultados obtenidos para los diversos subconjuntos de la población).

La segunda limitación relevante se refiere a la propia fiabilidad de los microdatos de renta y consumo contenidos en la EPF. Por una parte y al margen de los errores de muestreo, existen una serie de problemas asociados a la no respuesta y al tratamiento de los datos (procedimientos de imputación, elevación al año de registros semanales o mensuales, etc.) que pueden afectar a la fiabilidad, especialmente cuando se analizan los ingresos y consumos de los hogares individuales, más que los valores medios o agregados para grandes conjuntos de los mismos. Por otra parte no hay que olvidar el conocido problema de infradeclaración de los ingresos que resultan ser casi un $20 \%$ inferiores a las cifras de consumo estimadas a partir de la misma fuente.

Esta infradeclaración ha llevado a que la mayoría de los estudios sobre distribución en general, y sobre la situación económica de los diferentes grupos sociales en particular, se realicen a partir de las cifras de gastos. Esta práctica, que puede, justificarse con argumentos teóricos (el consumo corriente refleja mejor la renta permanente que las cifras de ingresos, más variables a lo largo del ciclo vital) no ayuda a despejar el camino hacia la investigación de una serie de aspectos fundamentales ligados a las fuentes de ingresos de la población, su nivel medio en 
los diversos colectivos y la forma en que estos ingresos se combinan dentro del hogar para formar el presupuesto familiar a cuyo cargo se realizan los consumos.

La ausencia de una investigación exhaustiva de las discrepancias entre los microdatos de renta y consumo recogidos en la EPF, obliga a tratar con cautela los resultados derivados de esta fuente, en tanto la explotación de otras bases de datos alternativas (como las de origen fiscal, cuyo desarrollo actual resulta muy prometedor) no ofrezca información adicional. Esta precaución resulta aún más necesaria si cabe en el caso de las personas mayores, ya que estudios anteriores han desvelado que es este grupo el que más varía su posición según se utilicen los datos de ingresos o los de gastos.

Otra limitación se refiere a la forma en que aparece organizada la información en la EPF, que es una encuesta sobre la renta y el consumo de los hogares, más que de los individuos. Aunque el hogar constituye una unidad de análisis relevante para conocer el nivel de vida de las personas que lo componen, obliga a asumir una serie de supuestos acerca de la distribución intrafamiliar de la renta y las escalas de equivalencia válidas para comprobar los hogares de distinto tamaño y composición. La estrategia que siguen los estudios que se basan en este tipo de datos suponen que: a) la renta se distribuye equitativamente dentro de cada hogar, y b) las ponderaciones dadas por 0,7 para los adultos adicionales y 0,5 para los niños (escala de la OCDE), constituyen una opción razonable. Así, el cociente de los ingresos totales del hogar entre la suma de las ponderaciones correspondientes, constituye la variable tomada como referencia para determinar la posición del hogar (y de sus miembros) en la escala de renta.

El hecho de que las principales clasificaciones sociodemográficas de los hogares se realicen atendiendo a las características del sustentador principal, lleva consigo una pérdida de información sobre los restantes miembros del hogar. Este problema se ve 
subsanado en la última versión de la EPF, que ofrece un amplio estudio de la situación de cada una de las personas que componen la unidad familiar.

La visión que los distintos especialistas pueden tener sobre el tema de la situación económica de los mayores, se mueve en dos polos diferentes y contradictorios. Los trabajadores sociales y las organizaciones benéficas y asistenciales tienen una visión un tanto catastrófica y negativa al respecto, por el contrario quienes trabajan con datos macroeconómicos consideran que es bastante buena o, que al menos ha mejorado en los últimos años.

Desde principios de los setenta existe una preocupación por la situación económica de los mayores reflejada en diversos estudios, sus pensiones, y la posible "carga" que puedan suponer para los gastos sociales. En 1976, apareció un trabajo realizado por Antonio de Pablo titulado: "Situación del grupo social de ancianos". "Dimensión y calidad de la respuesta internacional". Entre otras conclusiones, señalaba que en 1975 el millón y cuarto de personas en España que superan los sesenta y cinco años requiere importantes esfuerzos de la Administración para proveer a la atención de una edad que necesita mayores cuidados. El informe GAUR de 1975, sobre "La situación del anciano en España", publicado por la Confederación de las Cajas de Ahorro, señalaba el hecho de que ser jubilado equivale a ser un ser casi inútil, u miembro del grupo social que esta a expensas de las clases activas. Un informe de Cáritas de Vitoria publicado en los setenta: i... más allá de los sesenta y cinco años? Un reto a nuestra sociedad elaborado por el D.I.S, subrayaba como tema prioritario para mejorar la situación de los ancianos resolver de manera justa y equitativa el tema de las pensiones.

Que duda cabe que el fenómeno del envejecimiento demográfico ha afectado a lo económico, y la valoración que se ha hecho sobre la situación está marcada por una perspectiva economicista y por discursos excesivamente pesimistas acerca del 
sistema público de pensiones. Este discurso economicista tiende a ver al mayor más como un objeto de gasto social y como una carga para su familia, que ver los efectos favorables que puedan tener los mayores en el funcionamiento de la sociedad y de la economía.

En las últimas décadas se han producido dos fenómenos de manera simultánea, por un lado, un aumento en los gastos de protección social y de otro, el proceso de envejecimiento que ha tenido lugar en la población de estos países. Por ello se ha generalizado la idea de que el envejecimiento demográfico es uno de los motores fundamentales, aunque no el único del aumento de los gastos sociales. Para Guillemard (Guillemard, 1990); "el análisis más común asocia mecánicamente estos dos fenómenos para proponer una visión fatalista de las perspectivas de evolución de los sistemas de protección social", esta visión mecánica se refuerza todavía más con la visión negativa que recae sobre el proceso de envejecimiento demográfico. Pero hay otra serie de variables que intervienen en la evolución de los gastos sociales a veces más importantes que la edad de la población, la relación entre variables demográficas y otros ámbitos de la vida social no se puede reducir a un determinismo causal.

Como señala Julio Iglesias de Ussel (Iglesias de Ussel, 1988), un experto en la materia: "No se conoce bien en España el papel que están desempeñando los abuelos en la socialización de los nietos. Pero lo que sí es un hecho, es que una parte importante de la modernización de la sociedad y de la incorporación de la mujer al trabajo extradoméstico, se está realizando gracias al trabajo de los abuelos y abuelas; una especie de trabajo sumergido que permite el funcionamiento del hogar familiar. Ya la emigración de los años 60, hizo recaer la crianza de los hijos por los abuelos. Hoy la atención y cuidado de los niños, así como su traslado a los centros escolares, tareas domésticas, atención en situaciones de enfermedad, custodia de vacaciones (salidas 
de noche de viernes o sábado de sus hijos), recaen en gran parte sobre las abuelas y los abuelos en la sociedad española, cuando trabajan el padre y la madre".

\section{Análisis de la situación económica}

Sin hacer distinciones de género, edad, clase social, etc., la conclusión de más de un estudioso del tema es que la insolvencia económica de los mayores en España es un prejuicio estereotipado (Gil Calvo, 1993). Según esto, en España como en el resto de los países socialmente avanzados, se ha conseguido erradicar la pobreza a edades avanzadas. Puesto que los ingresos de los mayores de 64 años dependen en muy escasa proporción de las rentas personales del trabajo, los datos se referirán a los ingresos familiares.

Como hemos dicho con anterioridad, una vía indirecta para conocer el nivel económico de las personas mayores de 64 años es utilizando datos sobre la situación económica de los hogares, aportados por el INE mediante la Encuesta Continua de Presupuestos Familiares muy valiosa respecto al capítulo de ingresos y también incluye abundante información sobre los gastos. Sin embargo, subrayamos que pese a la gran utilidad de este tipo de información, a menudo la interpretación de los datos económicos es mucho menos deudora de la evolución de los ingresos personales que de la evolución de las estructuras familiares y la composición de los hogares.

El poder adquisitivo sigue siendo la base del nivel y el tipo de consumo que realizan los mayores, existiendo cierta heterogeneidad en los poderes adquisitivos. De acuerdo con un estudio del INSERSO (1995:164), un 60,4\% de los mayores en España cobra una pensión por debajo de las 65.0000 ptas. al mes, y solo un 9,6\% decían superar las 100.000 ptas. al mes. No hay que olvidar de que se trata de un segmento de población que suele infravalorar sus ingresos, por lo tanto como hamos 
comentado hay que dudar de la fiabilidad de estos resultados. Estos ingresos de las pensiones, son sólo una referencia sobre el poder adquisitivo y aún más sobre la calidad de vida, pues los mayores tienen los grandes gastos realizados (una vivienda equipada y en propiedad) e incluso algunos ahorros acumulados. Diferentes estudios muestran la coincidencia en la preocupación real de los mayores en llegar a final de mes. El ya citado estudio del INSERSO al realizar la pregunta; Con los ingreso totales de su hogar, llegan ud y su familia a finales de mes con mucha/bastante facilidad, con alguna/bastante/mucha dificultad, un 56,7\% dice llegar con dificultad a finales de mes, pero el dato se relativiza si lo desagregamos en las diferentes opciones; con alguna dificultad $(38,6 \%)$, con bastante dificultas $(12 \%)$, con mucha dificultad $(6,1 \%)$. Mientras que los que dicen llegar con facilidad representan el 41,5\%, que desagregado queda así: con mucha facilidad (5,7\%), con bastante facilidad (36,1\%). Estos datos son muy similares a los del estudio No 2072 del CIS, Apoyo informal a las personas mayores, 1993, se obtiene la siguiente tabla;

Tabla 1. Grado de dificultad de los mayores para llegar a finales de mes con los ingresos totales del hogar. España, 1993

\begin{tabular}{|l|c|c|}
\hline \multicolumn{1}{|c|}{ Grado de dificultad } & \multicolumn{2}{c|}{ Sexo } \\
\hline \hline & Varón & Mujer \\
\hline \hline Mucha facilidad & 7,5 & 3,9 \\
\hline \hline Bastante facilidad & 40,0 & 33,3 \\
\hline Alguna dificultad & 34,5 & 41,6 \\
\hline \hline Bastante dificultad & 10,2 & 13,3 \\
\hline \hline Mucha dificultad & 6,0 & 6,1 \\
\hline \hline NS/NC & 1,8 & 1,8 \\
\hline
\end{tabular}




\begin{tabular}{|l|c|c|}
\hline TOTAL & 100 & 100 \\
$(\mathrm{n})$ & $(1.035)$ & $(1.463)$ \\
\hline
\end{tabular}

Fuente: C.I.S. Apoyo informal a las personas mayores. Estudio no 2072, 1993

Un elemento a destacar en este estudio es que la mujer aparece como más pesimista, tienen menores porcentajes en las preguntas relacionadas con la facilidad de llegar a final de mes, y mayores en las relacionadas con la dificultad. Podríamos afirmar que en general existe una cierta percepción negativa por parte de los mayores sobre su situación económica. Esto podría tener repercusiones en el comportamiento económico, lo que podría llevar a controlar el gasto e incluso a que ahorren

Tabla 2. Ingresos individuales por pensiones e ingresos del hogar por unidad de consumo según sexo. (Españoles mayores de 64 años).

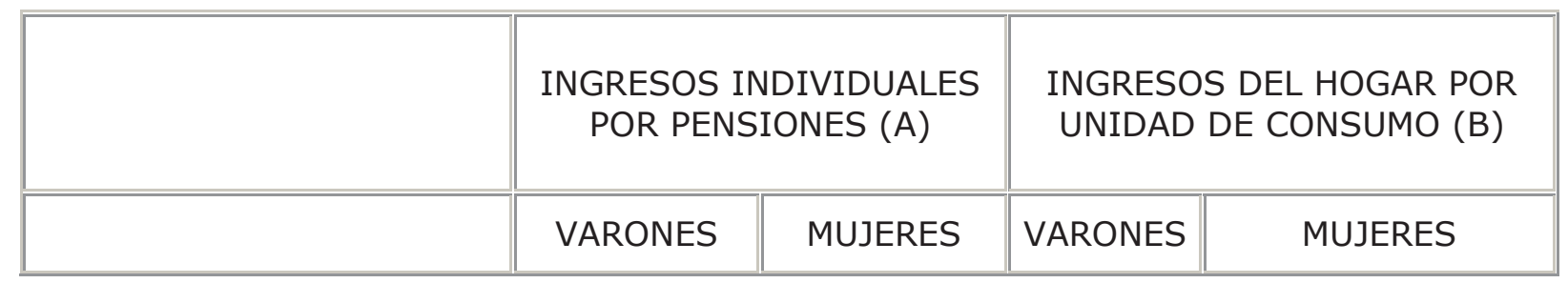




\begin{tabular}{|c|c|c|c|c|}
\hline \multicolumn{5}{|l|}{ Cantidad mensual: } \\
\hline Sin ingresos & $9.8 \%$ & $35.2 \%$ & $.0 \%$ & $.0 \%$ \\
\hline menos de 20.000 ptas. & $.8 \%$ & $2.0 \%$ & $.4 \%$ & $.8 \%$ \\
\hline \multirow{2}{*}{ de 20.000 a 40.000 ptas. } & $8.1 \%$ & $24.2 \%$ & $15.3 \%$ & $14.4 \%$ \\
\hline & $46.2 \%$ & $32.0 \%$ & $31.2 \%$ & $31.0 \%$ \\
\hline de 40.000 a 60.000 ptas. & $13.6 \%$ & $3.5 \%$ & $25.4 \%$ & $25.6 \%$ \\
\hline de 60.000 a 80.000 ptas. & $8.2 \%$ & $1.6 \%$ & $12.8 \%$ & $13.0 \%$ \\
\hline de 80.000 a 100.000 ptas. & $13.4 \%$ & $1.5 \%$ & $14.9 \%$ & $15.1 \%$ \\
\hline más de 100.000 ptas & $(100 \%)$ & $(100 \%)$ & $(100 \%)$ & $(100 \%)$ \\
\hline \multicolumn{5}{|c|}{ Fuente: I.N.E., Encuesta de Presupuestos Familiares 1990-91 } \\
\hline \multicolumn{5}{|c|}{$\begin{array}{l}\text { (B).- Incluye ingresos monetarios y no monetarios (autoconsumo, alquiler } \\
\text { imputado a la vivienda, etc.). }\end{array}$} \\
\hline
\end{tabular}

Si tan sólo atendemos a la distribución de los ingresos individuales por pensiones de los mayores de 64 años, esta es, claramente favorable a los varones. El 35\% de las mujeres carece de ingresos propios y solo el 9.6\% de los varones. La mayoría de ellas poseen ingresos inferiores a 60.000 ptas., mientras que un 81,6\% de los hombres posee ingresos iguales o superiores a 60.000 ptas.

En cambio si tomamos como punto de referencia los ingresos del hogar en que viven, esta distribución por sexos se torna muy igualitaria, concentrándose la mayoría de los hogares (algo más del 50\%) en el tramo de ingresos de 40.000 a 80.000 ptas.

La convivencia de las mujeres ancianas con sus maridos, con sus hijos o con otras personas compensa generalmente su peor situación en cuanto a ingresos 
individuales. Con todo, no se puede suponer que esto anule la mayor vulnerabilidad de las mujeres, pues la situación económica de muchas de ellas es de clara dependencia económica. Cabe señalar, que utilizar los ingresos del hogar como indicador de la posición económica de alguno de sus miembros, supone asumir que la distribución de los ingresos es homogénea dentro del grupo familiar. Como dice Fernández Viguera (1.995: 77), refiriéndose a las mujeres de cualquier edad, "la dependencia económica del marido, que en ocasiones es vivida como temporal, se convierte en permanente y sin vuelta y empobrece a las mujeres de forma encubierta, haciéndose visible en los procesos de separación y divorcio". En la vejez, sin embargo, hay razones para poner en duda esta mayor vulnerabilidad de la mayoría de las mujeres, sobre todo si es verdad que ellas tienden a adquirir un mayor protagonismo y poder en el ámbito doméstico.

Tabla ${ }^{0} 3$. Tipos de pensiones según el sexo.

\begin{tabular}{|l|c|c|}
\hline \multicolumn{1}{|c|}{} & Varones & Mujeres \\
\hline Pensión jubilación propia & $89,9 \%$ & $33,5 \%$ \\
\hline $\begin{array}{l}\text { Pensión jubilación del } \\
\text { cónyuge }\end{array}$ & $5,7 \%$ & $38,8 \%$ \\
\hline Pensión de viudedad & $0,3 \%$ & $28,5 \%$ \\
\hline Fuente: INSERSO $\mathbf{1 . 9 9 5}$ & & \\
\hline
\end{tabular}

En consonancia con la tabla anterior, el 89,9\% de los varones son los que cuentan con una pensión de jubilación propia, mientras que las mujeres dependen de manera bastante significativa de la pensión de jubilación del cónyuge o de la de viudedad. En relación con la cuantía de estas pensiones, la limitación en la pensión máxima, la elevación en la mínima, y la mejora notable en la situación económica de ciertos tipos de pensiones (como por ejemplo las de las viudas: $A B C, 3 / 11 / 98$ ), van a tender a igualar la situación endógena de los mayores, acortando las diferencias en la cuantía de las pensiones entre los sexos. 
Un estudio llevado a cabo por el Centro de Investigaciones Sociológicas (CIS, 1993), un poco más reciente, viene a reforzar los datos expuestos con anterioridad, en la tabla se muestran tan solo los ingresos mensuales según el sexo. Mientras que en la tabla anterior la mayoría percibía unos ingresos situados en el tramo de 40.000 a 60.000 ptas., esta afina un poco más y sitúa a la media de la población entre 45.000 y 55.000 pesetas mensuales $(29,0 \%)$ y en segundo lugar entre 55.000 y 65.000 ptas. Teniendo en cuenta el sexo, los varones siempre tienen porcentajes mayores en las cantidades superiores a 55.000 ptas., siendo significativo el dato de que un $14,6 \%$ de los varones frente a solo un $6 \%$ de mujeres, tienen ingresos superiores a 100.000 ptas.

Tabla 4. Ingresos mensuales de los mayores según sexo. España, 1993

\begin{tabular}{|c|c|c|c|}
\hline Ingresos & Varones & Mujeres & Media \\
\hline \hline 25.000 & 0,5 & 2,9 & 1,9 \\
\hline $25.000-35.000$ & 2,3 & 7,7 & 5,4 \\
\hline $35.000-45.000$ & 5,0 & 13,5 & 10,0 \\
\hline $45.000-55.000$ & 24,9 & 31,8 & 29,0 \\
\hline $55.000-65.000$ & 17,9 & 11,4 & 14,1 \\
\hline $65.000-75.000$ & 8,0 & 5,3 & 7,3 \\
\hline $75.000-85.000$ & 9,3 & 5,8 & 8,7 \\
\hline \hline $85.000-100.000$ & 9,6 & 8,1 & 9,6 \\
\hline \hline$>100.000$ & 14,6 & 6,0 & $\mathbf{2 0 7 2}$ \\
\hline \hline Fuente: CIS: Apovo informal a las personas mayores. Estudio no & \\
\hline \hline
\end{tabular}




\subsection{Evolución de los ingresos y gastos por edad.}

La única forma que existe para abordar esta cuestión, como hemos mencionado con anterioridad, es de manera indirecta utilizando los datos sobre la evolución de la situación económica de los hogares aportados por el INE mediante la Encuesta Continua sobre Presupuestos Familiares.

Si se tiene en cuenta el incremento anual de todos los ingresos y los gastos realizados por el conjunto de familias en España, tomando como base su situación en 1985, y haciendo una distinción de los diferentes tramos de edad del cabeza de familia, el resultado es bastante significativo. Es en los hogares en que el cabeza de familia supera los 54 años es donde los ingresos y los gastos tienen un crecimiento mayor al del conjunto nacional, ello es coincidente en los datos de los tres años disponibles, 1989, 1990 y 1991. Aún más, los crecimientos máximos de ambos conceptos se producen en los hogares encabezados por mayores de 64 años. Se obtienen resultados similares si se discrimina en función de la actividad del cabeza de familia. De nuevo, el mayor incremento se produce, en los tres años, en los hogares encabezados por jubilados, frente a los de los ocupados, que se sitúan cercanos a la media nacional, y a los hogares encabezados por desempleados y otros inactivos no jubilados, que presentan un crecimiento de gastos e ingresos inferior al conjunto.

Tabla 5. Incremento $(\%)$ sobre el año base $(1985=1000)$ del total anual ingresado y gastado por todas las familias españolas.

\begin{tabular}{|c|c|c|c|c|c|c|}
\hline & \multicolumn{2}{|c|}{1989} & \multicolumn{2}{|c|}{1990} & \multicolumn{2}{|c|}{1991} \\
\hline $\begin{array}{l}\text { Cabeza de } \\
\text { Familia }\end{array}$ & Ingreso & Gasto & Ingreso & Gasto & Ingreso & Gasto \\
\hline Ocupados & 59,6 & 50,8 & 79,8 & 67,4 & 98,4 & 83,9 \\
\hline Jubilados & 73,1 & 61,7 & 107,5 & 93,4 & 136,6 & 111,4 \\
\hline $\begin{array}{l}\text { Media } \\
\text { Nacional }\end{array}$ & 57,3 & 47,8 & 79,4 & 66,3 & 100,9 & 83,7 \\
\hline
\end{tabular}

Fuente: INE, Encuesta Continua de Presupuestos Familiares. 
Estos datos son mucho más elocuentes si se tiene en cuenta la evolución del desempleo, y muestran con claridad los efectos del cambio en la estructura de la población y el aumento consecuente del peso de mayores de 64 años en el conjunto. Los efectos del cambio de estructura por edades, son bastante complejos. El ritmo de creación de nuevos hogares, también inferior, es otra de las causas del crecimiento de la parte de ingresos y gastos familiares correspondientes a los encabezados por mayores de 64 años. Los hijos en las primeras edades activas, aquellos que no están creando nuevos hogares, sólo pueden contribuir de manera muy limitada, sin embargo, a aumentar los ingresos y gastos de sus antiguos hogares, puesto que se lo impide el elevado desempleo juvenil y la mayor dedicación a la mejora de la cualificación mediante los estudios. En cambio, los que una vez finalizado el periodo de formación se encuentran ocupados y continúan viviendo con sus padres (situación que se da cada vez con mayor frecuencia) sí deben de tener consecuencias significativas en el aumento del conjunto de ingresos y gastos de los hogares encabezados por personas de edades maduras-avanzadas.

Por lo tanto, el incremento en la proporción de mayores de 64 años solo explica una parte del superior ritmo de crecimiento de los ingresos y gastos del conjunto de los hogares donde viven los jubilados, hay que aclarar qué parte debe atribuirse a los cambios en la estructura de los hogares y qué parte corresponde a variaciones reales en el poder adquisitivo de los individuos de cada grupo de edad.

Tabla 6. Incremento $(\%)$ sobre el año base $(1985=1000)$ del total anual ingresado y gastado por todas las familias españolas, según el tipo de hogar.

\begin{tabular}{|c|c|c|c|c|c|c|}
\hline Tipo de hogar & \multicolumn{2}{|c|}{1989} & \multicolumn{2}{|c|}{1990} & \multicolumn{2}{|c|}{1991} \\
\hline & Ingreso & Gasto & Ingreso & Gasto & Ingreso & Gasto \\
\hline Unipersonal $<65$ & 9,8 & 2,1 & 34,0 & 25,0 & 60,9 & 52,4 \\
\hline
\end{tabular}




\begin{tabular}{|c|c|c|c|c|c|c|}
\hline Unipersonal > 64 & 87,6 & 76,5 & 127,5 & 124,3 & 161,1 & 157,4 \\
\hline Pareja sin hijos & 50,1 & 51,9 & 88,6 & 88,5 & 113,7 & 110,7 \\
\hline Pareja con un hijo & 59,8 & 54,2 & 75,8 & 67,6 & 82,9 & 71,1 \\
\hline $\begin{array}{l}\text { Pareja con dos } \\
\text { hijos }\end{array}$ & 42,2 & 38,6 & 55,7 & 47,2 & 58,5 & 47,2 \\
\hline Media nacional & 57,3 & 47,8 & 79,4 & 66,3 & 100,9 & 83,7 \\
\hline \multicolumn{7}{|c|}{$\begin{array}{l}\text { Fuente: INE, Encuesta Continua de Presupuestos Familiares. Extraída de Gil } \\
\text { Calvo, op. cit. pg. } 13\end{array}$} \\
\hline
\end{tabular}

De acuerdo con la tabla anterior, en esta puede comprobarse que es el conjunto de los hogares ocupados por mayores de 64 años que viven solos los que muestran un mayor crecimiento en el conjunto de los ingresos familiares y, sobre todo, en el de los gastos. Es todo lo contrario de lo que ocurre con los hogares de este tipo ocupados por personas de menos de 65 años, que no se acercan siquiera al crecimiento medio nacional. El otro tipo de hogares que se sitúa por debajo de dicho crecimiento medio es el de los hogares más numerosos, es decir, las familias nucleares con más de un hijo, que aquí solo aparecen las parejas con dos hijos. Por lo tanto, son los hogares de tamaño reducido, con la excepción de los solitarios menores de 65 años, los que presentan incrementos importantes.

Cabe recordar, que el tamaño medio de los hogares españoles está disminuyendo rápidamente en los últimos años, y que dicho factor, junto a la evolución de sus estructuras del hogar, se correlaciona con el volumen total de ingresos y gastos que supone cada tipo de hogar. Por ello, lo que haremos a continuación será examinar la evolución de los ingresos y gastos por persona en los distintos tipos de familia.

Tabla 7. Proporción (\%) que guardan los ingresos y gastos por persona respecto al promedio del total nacional

\begin{tabular}{|c|c|c|c|c|c|c|}
\hline & \multicolumn{2}{|c|}{1989} & \multicolumn{2}{|c|}{1990} & \multicolumn{2}{|c|}{1991} \\
\hline & Ingreso & Gasto & Ingreso & Gasto & Ingreso & Gasto \\
\hline Total nacional & 100,0 & 100,0 & 100,0 & 100,0 & 100,0 & 100,0 \\
\hline
\end{tabular}




\begin{tabular}{|c|c|c|c|c|c|c|}
\hline \multicolumn{7}{|l|}{$\begin{array}{l}\text { Edad cabeza de } \\
\text { familia }\end{array}$} \\
\hline $25-34$ & 103,4 & 103,0 & 105,3 & 103,4 & 101,9 & 99,4 \\
\hline $35-44$ & 92,6 & 96,0 & 94,0 & 97,4 & 92,6 & 97,4 \\
\hline $45-54$ & 96,0 & 97,8 & 93,0 & 95,7 & 96,0 & 98,3 \\
\hline $55-64$ & 107,1 & 106,7 & 107,4 & 104,8 & 105,2 & 105,3 \\
\hline 65 y más & 105,6 & 98,9 & 106,5 & 101,6 & 108,0 & 99,5 \\
\hline \multicolumn{7}{|l|}{$\begin{array}{l}\text { Actividad cabeza de } \\
\text { familia }\end{array}$} \\
\hline Ocupado & 102,5 & 103,3 & 102,0 & 102,6 & 101,4 & 103,0 \\
\hline Jubilado & 100,4 & 96,5 & 100,5 & 97,7 & 101,8 & 96,2 \\
\hline \multicolumn{7}{|l|}{ Tipo de hogar } \\
\hline Unipersonal < 65 & 192,7 & 181,4 & 195,4 & 186,9 & 189,7 & 186,8 \\
\hline Unipersonal > 64 & 126,0 & 114,1 & 130,0 & 124,8 & 135,1 & 131,6 \\
\hline Pareja sin hijos & 121,9 & 120,9 & 125,2 & 124,1 & 122,4 & 121,6 \\
\hline Pareja con un hijo & 125,9 & 125,6 & 118,6 & 118,6 & 116,4 & 115,8 \\
\hline $\begin{array}{l}\text { Pareja con dos } \\
\text { hijos }\end{array}$ & 96,3 & 102,3 & 97,5 & 101,8 & 91,2 & 95,2 \\
\hline
\end{tabular}

En esta tabla, los datos nos revelan de manera más clara como los ingresos por persona en los hogares de los mayores de 64 años ha crecido de un modo que supera proporcionalmente al promedio nacional. Si el criterio que tomamos son los tipos de hogar, dejando a parte los unipersonales cuyos ocupantes tiene una edad inferior a los 65 años, los solitarios mayores de 64 años se sitúan también por encima de los ingresos medios por persona del resto de tipos de hogar, e incluso de los gastos, de manera muy significativa en 1991. Como manifiesta Preston en España se produce el mismo fenómeno de inversión entre los niveles de bienestar de las diferentes edades:

"Son los hogares cuyos cabezas de familia superan los 54 años, que ya no tienen mayoritariamente hijos convivientes o dependientes, quienes ostentan el liderazgo de ingreso personal y de gasto personal. En cambio, las familias cuyos cabezas tienen edades entre 35 y 54 años, en las que conviven más hijos dependientes, son las que proporcionan menores proporciones relativas de gasto y de ingreso por persona, sensiblemente inferiores al promedio" (Preston, 1984). 
El tratar con datos relativos a los ingresos familiares por persona tiene el inconveniente de obviar las economías de escala que subyacen en la organización familiar. Es evidente que para conseguir el mismo nivel de bienestar económico no es necesario que los ingresos del hogar unipersonal se multipliquen aritméticamente por el número de integrantes de un hogar con más personas (no es necesario que cada uno adquiera o alquile una vivienda, tenga una lavadora o prepare la comida en una cocina diferente).

Puesto que los ingresos de las personas que viven solas y tienen menos de 65 años son sólo 1,4 veces superiores a la de los viejos que viven en la misma situación, todo apunta a que es la unión de dos personas que aportan ingresos propios la que constituye la pauta general a la hora de formar un nuevo hogar y tener hijos. Lo que se está observando en realidad en la anterior tabla es el resultado de la creciente contribución de la mujer a los ingresos familiares, no sólo en los de nueva formación, sino incluso en aquellos en que inicialmente sólo trabajaba el hombre fuera del hogar.

En lo que se refiere a la relación entre ingreso y gasto en cada una de las categorías de la tabla anterior, puede constatarse que el ahorro es superior en las edades avanzadas, que no llegan a agotar su capacidad de consumo, mientras que ocurre todo lo contrario en las edades más jóvenes, en las que hay que hablar más bien del endeudamiento que del ahorro. Si la observación se hace sobre los tipos de hogar, son los adultos con familiares a cargo los que gastan por encima de lo que ingresan, mientras que los menos endeudados son los hogares unipersonales. Pero cuando se habla del gasto en las diferentes edades, no sólo debe considerarse su cuantía, sino también sus características. Los hogares unipersonales, ya sean de jóvenes o de viejos, no se encuentran embarcados en el tipo de gastos que podría considerarse de inversión, especialmente de inversión en patrimonio. En cambio, es seguro que una elevada proporción de parejas con hijos, precisamente por el tipo de hogares que presenta un mayor endeudamiento, está amortizando préstamos para la adquisición 
de una vivienda. Debe considerarse, además, que al margen de la intensidad del endeudamiento derivada de la constitución de un nuevo hogar, los hijos introducen nuevas posibilidades de consumo vedadas a quienes no los tienen. Incluso sin la presencia de hijos en el hogar, hay que considerar que la estructura de consumo es diferente entre jóvenes y ancianos, aunque solo sea por la aparición de nuevos productos dirigidos a edades específicas, uno de los efectos más visibles de las nuevas economías postindustriales.

Cabe recordar también que desde la segunda guerra mundial, el ahorro en el mundo occidental se encuentra determinado constantemente por los efectos de la inflación, como viene recordando sistemáticamente Alfred Sauvy en su obra "Los límites de la vida humana" de 1964, cuando trata los determinantes del ahorro entre los ancianos. Este factor, unido a la extinción de las rentas derivadas del trabajo, y a la incertidumbre sobre los años de vida que les quedan, crea en estas personas una necesidad de seguridad cubierta por la restricción del consumo. 


\section{La renta de las personas mayores}

El rasgo principal que diferencia a este grupo del resto de la población es el origen de los ingresos, que ya no proceden tanto del mercado de trabajo como de los sistemas públicos de transferencias, basados en mayor o menor medida en las cotizaciones realizadas durante la vida activa, y del ahorro acumulado a lo largo del periodo anterior. Esta diferenciación en las fuentes de ingresos contribuye a que los factores que inciden en la evolución del nivel medio y distribución de la renta en el caso de los jubilados no sean los mismos que afectan a los ingresos de la población en edad de trabajar.

Sin negar la importancia que tienen las pensiones para este colectivo, sus ingresos finales dependen de otras fuentes de renta, como pueden ser el mantenimiento de ciertos vínculos con la actividad laboral, la gestión privada de la renta acumulada a lo largo del ciclo vital, ya sea a través de fondos de pensiones u otro tipo de rentas de la propiedad, o cualesquiera otros tipos de ingresos. Otro flujo de renta de las personas mayores que resulta difícil de valorar, es el realizado por los descendientes $\mathrm{u}$ otros miembros de la familia, ya sea en formas de transferencias directas, ya mediante la prestación de determinados servicios. Si tradicionalmente en España el papel de la familia había sido importante en el mantenimiento de las rentas de las personas mayores, en la etapa reciente se da el proceso inverso en lo que se refiere a la contribución económica de las personas mayores al sostenimiento de sus descendientes, abarcando en algunos casos a integrantes de dos generaciones.

Según datos del CIS, solo el 11\% de las personas mayores españolas contaba con fuentes de ingresos distintas de las pensiones, cifras muy lejanas a las de otros países como Estados Unidos o Inglaterra, en los que la Seguridad Social proporcionaba tan sólo cerca de la mitad de los ingresos de este colectivo. Según la EPA un 2,7\% de la población mayor de 64 años desempeñaba una actividad laboral. Los datos de la EPF 
ofrecen datos similares, con un 3,2 \% en el caso de los varones y un 1,6\% en lo que respecta a las mujeres en 1990, aunque hay que resaltar que estos porcentajes referidos a la población en hogares sustentados por personas mayores, no son plenamente comparables con los de la EPA. Un porcentaje significativo de los mayores que trabajan lo hacen para complementar los ingresos procedentes de otras fuentes de renta, lo que puede apreciarse a través de los datos del empleo a tiempo parcial. Según un estudio reciente Toharia (1993), la incidencia del trabajo a tiempo parcial es mucho mayor en el segmento de asalariados mayores de 64 años que en el resto de trabajadores. Los datos parecen poner de manifiesto un proceso ya sucedido con anterioridad en otros países, se refiere al carácter progresivamente residual del mercado de trabajo como fuente generadora de ingresos para las personas mayores. A ello ha contribuido la política de ceses voluntarios en forma de jubilaciones anticipada, propiciada por la necesidad de reconversión de la estructura productiva ante el declive de determinados sectores industriales. También el deterioro del mercado de trabajo y la concentración del desempleo de larga duración en trabajadores con la edad más alta, ha obligado a los poderes públicos a instrumentar planes de jubilaciones anticipadas.

En este cuadro se observa, que solo alrededor de un 15\% de las personas mayores afirman depender total o parcialmente, en términos económicos de algún familiar, tanto en 1.992 como en 1995, por lo tanto la población mayor depende mayoritariamente de sus ingresos.

Tabla 8. Dependencia económica de los mayores de 65 años de algún familiar

\begin{tabular}{|c|c|c|c|c|}
\hline & \multicolumn{2}{|c|}{ Total } & \multicolumn{2}{|c|}{ Mayores } \\
\hline & 1.992 & 1.995 & 1.992 & 1.995 \\
\hline TOTAL & 1.200 & 1.200 & 196 & 220 \\
\hline Sí, totalmente & $4 \%$ & $7 \%$ & $6 \%$ & $7 \%$ \\
\hline Sí, en parte & 10 & 10 & 7 & 8 \\
\hline No & 86 & 82 & 86 & 83 \\
\hline
\end{tabular}




\begin{tabular}{|l|l|l|l|}
\hline NS/NC & 1 & 1 & $*$ \\
\hline
\end{tabular}

Fuente: La sociedad Española 1994-95. Amando de Miguel.

\section{Consumo de los hogares cuyo sustentador principal tiene más de 65 años.}

En la década 1981-91, el número de hogares cuyo sustentador principal tenía 65 años o más, pasó de representar el 20,4\% al 25,1\%. Una característica de los hogares cuyo sustentador principal tiene 65 años o más es que sus necesidades, y por lo tanto sus hábitos de consumo difieren de los del resto de los hogares. Cada tipo de hogar, por características socioeconómicas, nivel de estudios del cabeza de hogar, nivel de ingresos, etc., tiene diferentes hábitos de consumo. Tales hábitos de consumo pueden conocerse mediante los gastos de las familias por grupos de bienes contenidos en la EPF.

Sin embargo en lo que se refiere a las personas mayores, una parte de sus necesidades viene satisfecha por servicios tales como prestaciones sanitarias o servicios sociales, y no por el gasto directo de los hogares en los bienes y servicios necesarios para su satisfacción. El gasto equivalente de dichos servicios públicos no está registrado en los gastos reflejados en la encuesta.

Tabla 9. Gasto de los hogares por grupos de bienes

\begin{tabular}{|c|c|c|c|c|}
\hline & \multicolumn{2}{|c|}{ Todos los hogares } & \multicolumn{2}{|c|}{$\begin{array}{c}\text { Hogares con sustentador principal de } 65 \text { y más } \\
\text { años. }\end{array}$} \\
\hline & 1981 & 1991 & 1981 & 1991 \\
\hline $\begin{array}{l}\text { Gasto medio por } \\
\text { persona (ptas. } \\
\text { corrientes) }\end{array}$ & 237.694 & 741.742 & 227.400 & 726.845 \\
\hline $\begin{array}{l}\text { Gasto medio por hogar } \\
\text { (ptas. corrientes) }\end{array}$ & 879.251 & 2.527 .105 & 517.225 & 1.624 .665 \\
\hline \multicolumn{5}{|c|}{ Distribución del porcentaje del gasto por hogar } \\
\hline $\begin{array}{l}\text { 1. Alimentos, bebidas } \\
\text { y tabaco }\end{array}$ & 31,9 & 24,6 & 37,8 & 28,3 \\
\hline 2. Vestido y calzado & 8,5 & 9,5 & 7,4 & 8,4 \\
\hline $\begin{array}{l}\text { 3. Vivienda, } \\
\text { calefacción y } \\
\text { alumbrado }\end{array}$ & 18,8 & 22,6 & 23,6 & 29,3 \\
\hline
\end{tabular}




\begin{tabular}{|c|c|c|c|c|}
\hline $\begin{array}{l}\text { 4. Mobiliario, menaje y } \\
\text { conservación vivienda }\end{array}$ & 7,5 & 5,5 & 7,4 & 5,5 \\
\hline $\begin{array}{l}\text { 5. Servicios médicos y } \\
\text { gastos sanitarios }\end{array}$ & 2,4 & 2,6 & 2,9 & 2,7 \\
\hline $\begin{array}{l}\text { 6. Transportes y } \\
\text { comunicaciones }\end{array}$ & 13,6 & 12,2 & 8,1 & 7,6 \\
\hline $\begin{array}{l}\text { 7. Esparcimiento, } \\
\text { espectáculo, } \\
\text { enseñanza y cultura }\end{array}$ & 6,7 & 6,1 & 3,7 & 3,5 \\
\hline $\begin{array}{l}\text { 8. Otros bienes y } \\
\text { servicios }\end{array}$ & 8,4 & 12,9 & 6,9 & 10,2 \\
\hline 9. Otros gastos & 2,2 & 4,2 & 2,3 & 4,4 \\
\hline
\end{tabular}

Fuente: INE, Encuesta de Presupuestos Familiares; 1980/1981 y 1990/ 1991.

Los hogares formados por personas de más edad suelen coincidir con las fases de "nido vacío II" (matrimonio sin hijos que convivan con ellos) "nido vacío III" (matrimonio con uno de los cónyuges jubilado), y supérstite solitario, gastan más que la media en los grupos: G1 (alimentación, bebidas y tabaco), G3 (vivienda, calefacción y alumbrado) y G5 (servicios médicos y gastos sanitarios).. El gasto sanitario evoluciona a lo largo de la vida siguiendo una pauta en forma de $\mathrm{U}$, es decir, un consumo muy intenso en los primeros años de la vida y en la vejez. Hay una serie de factores que provocan que este gasto sanitario sea excesivo, ya que tienen que ver en mayor medida con una deficiente disposición de los recursos que con el efecto del proceso de envejecimiento; como la carencia de estructuras alternativas de menor coste y mas adecuadas para el cuidado sanitario de los ancianos como los centros de día, unidades geriátricas o residencias asistidas. El gasto farmacéutico de los pensionistas es 8,5 veces superior al de los activos, y el número de recetas es más de 5 veces superior, a lo que se añade que el coste por receta es inferior en 1,56 veces. Este elevado consumo se debe a varios factores como la automedicación, el exceso de medicamentos prescrito por los médico y también al uso fraudulento que realizan sus familiares y llegados que no pertenecen a esa categoría de población.

También cabe destacar que las alteraciones sufridas por la distribución del gasto para el conjunto de los hogares entre 1980-91, que consisten en una disminución de la 
participación de los gastos de alimentación y mobiliario ( 1 y 4 ) y el aumento del gasto en vestido, vivienda, otros bienes y servicios y otros gastos (partidas 2,3, 8 y 9), se reproducen para los hogares cuyo sustentador principal tiene 65 o más años. Esta invarianza del cambio en la distribución del gasto por el tipo de hogar revela que los hogares de

las personas de edad no son ajenos a los cambios en la estructura del gasto inducidos por el aumento progresivo del nivel de vida aunque dependan más que los demás hogares de ciertos tipos de gastos. En resumen, los hogares encabezados por personas de edad, comparten los cambios sociales en la estructura de consumo, lo cual refleja el dinamismo de sus miembros.

\section{El ahorro para la vejez.}

La implantación paulatina de los sistemas públicos de previsión, está siendo sustituida por una proliferación de los planes privados de pensiones para la vejez, como consecuencia de la modificación de la llamada Sociedad de Bienestar en España. Cada vez gana más importancia el término preparación para la jubilación, no sólo económica, sino también de carácter psicosocial, para ello vamos a analizar un sencillo indicador de tipo económico: el ahorro anterior a la jubilación, que realizan los mayores, con el propósito de tener un cierto respaldo económico durante la última etapa de la vida.

El dinero (ahorro) aporta a los mayores una gran seguridad, lo entienden como un seguro para sacarles de apuros en caso de caer enfermos (para garantizarse una asistencia, bien por canales formales o informales) y también para hacer frente a los imprevistos que puedan ocurrir en el seno de la propia familia. 
En los datos obtenidos, algo más de la mitad (56\%) de los españoles hoy jubilados han estado ahorrando para enfrentarse a la vejez. Sin embargo esta proporción si desagregamos por sexo, es superior en el caso de los varones hasta alcanzar a casi dos terceras partes (64\%) de los mismos, frente a menos de la mitad (44\%) de las mujeres. Esta pauta de las mujeres, no creemos que se deba a una actitud de menor previsión frente a la vejez, sino (a parte de otras razones) a las circunstancias de menor remuneración en la que se han visto envueltas las mujeres, frente a los varones, a pesar a veces de desempeñar tareas de idéntica categoría laboral. No obstante estos porcentajes tenderán a ir en aumento debido a que las nuevas generaciones de jubilados, van a disfrutar en menor medida de las pensiones públicas y tendrán que garantizarse otros medios para alcanzar una cierta seguridad económica en el momento de la jubilación (planes privados, ahorros, inversiones, seguros de pensiones...). La diferencia citada anteriormente en relación a los sexos se mantiene en casi todas las categorías de edad, estado civil, clase social y estudios. Si bien, esta distancia se acorta perdiendo significatividad al comparar el comportamiento de los solteros y las solteras, o a los varones o mujeres sin estudios primarios. Respecto al estado civil, las mujeres jubiladas solteras han sido más previsoras, con diferencia significativa, que las casadas y las viudas, ya que al no depender lo económico del cónyuge ellas mismas se hacen cargo. En cambio, no ocurre lo mismo, con los solteros respecto de los casados, dado que los porcentajes de personas que ahorran son similares.

Tabla 10.Porcentaje de jubilados que ahorraron para la vejez

\begin{tabular}{|c|c|c|c|c|}
\hline & & VARONES & MUJERES & TOTAL \\
\hline \multirow{2}{*}{ EDAD } & -75 & 64 & 43 & 56 \\
\hline & +75 & 62 & 45 & 56 \\
\hline \multirow{3}{*}{ ESTADO CIVIL } & SOLTEROS & 65 & 58 & 60 \\
\hline & CASADOS & 66 & 41 & 61 \\
\hline & VIUDOS & 51 & 40 & 44 \\
\hline \multirow[t]{2}{*}{ CLASE SOCIAL } & ALTA & 67 & 50 & 61 \\
\hline & MEDIA & 68 & 48 & 60 \\
\hline
\end{tabular}




\begin{tabular}{|l|l|l|l|l|}
\hline \multicolumn{1}{|c|}{} & BAJA & 60 & 40 & 52 \\
\hline \multirow{3}{*}{ NIVEL DE ESTUDIOS } & + PRIMARIOS & 61 & 43 & 54 \\
\cline { 2 - 5 } & = PRIMARIOS & 69 & 46 & 60 \\
\cline { 2 - 5 } & - PRIMARIOS & 46 & 39 & 43 \\
\hline TOTAL & 64 & 44 & 56 \\
\hline \hline
\end{tabular}

Fuente: La Sociedad Española, 1994-95. Amando de Miguel.

Otra característica que explica que unas personas mayores sean más previsoras que otras, es la edad de jubilación, es lógico pensar que cuando el retiro laboral se adelanta hay menos tiempo para ahorrar (Amando de Miguel, 1994-95). La influencia de esta circunstancia es tal que acaba anulando las diferencias entre varones y mujeres cuando las jubilaciones se producen a los 65 años o después de esta edad. También influye el hecho de que en las jubilaciones postergadas se van concentrando los trabajadores con los mejores puestos y con una mayor capacidad económica. Pero no se trata solo de una cuestión de ocupación, sino de tiempo real necesario para ir generando (además de otros ahorros para otros gastos más inmediatos) el ahorro específico de la vejez.

Como se deja notar, ya desde principios de los setenta existe una preocupación por la situación económica de los mayores reflejada en diversos estudios, sus pensiones, y la posible "carga" que puedan suponer para los gastos sociales.

\section{El consumo tras la jubilación}

La vejez como otras etapas de la vida se caracteriza por una concurrencia de transiciones, sin embargo estas tienen un signo bien distinto. Mientras que en la edad adulta se produce el paso al propio hogar, la constitución de una familia, el aumento del consumo en todo tipo de bienes (casa, coche, ocio) y la recepción de la herencia, en la vejez concurren la reducción del consumo, el abandono del hogar por los hijos, el enviudamiento y la transmisión de la herencia. En la siguiente tabla aparece 
reflejada la concurrencia o no de las circunstancias mencionadas anteriormente tras la jubilación, apareciendo las frecuencias totales y para cada sexo.

Tabla 11. Circunstancias ocurridas tras la jubilación

\begin{tabular}{|c|c|c|c|}
\hline $\begin{array}{l}\text { (1.993) } \\
\% \text { verticales (múltiples) }\end{array}$ & & le jubilad & \\
\hline $\begin{array}{l}\text { Circunstancias ocurridas tras } \\
\text { la jubilación }\end{array}$ & Varones & Mujeres & Total \\
\hline $1^{\circ}$ Reducción del consumo & 43 & 41 & 43 \\
\hline $\begin{array}{l}2^{\circ} \text { Transmisión de la } \\
\text { herencia }\end{array}$ & 21 & 27 & 23 \\
\hline $3^{\circ}$ Enviudamiento & 15 & 30 & 20 \\
\hline $\begin{array}{l}4^{\circ} \text { Solicitud de ayuda } \\
\text { institucional }\end{array}$ & 7 & 9 & 7 \\
\hline $5^{\circ}$ Venta de la casa & 2 & 3 & 2 \\
\hline
\end{tabular}

La lista se encuentra encabezada por la reducción del consumo de algunos productos, constatada por cuatro de cada diez personas mayores, sigue la transmisión de la herencia y el enviudamiento, hechos que han afectado a una quinta parte de los encuestados. Estas proporciones varían según el sexo, siendo superior en todas las circunstancias para las mujeres.

En lo que se refiere al consumo de los mayores, este no depende tan solo del poder adquisitivo, hay factores cultural-generacionales, históricos, familiares e individuales que también influyen. Como es sabido el nivel de vida está articulado en torno a procesos individuales y familiares, que se estructuran en torno al poder adquisitivo y a los hábitos de consumo adquiridos. El mayor tiene unos hábitos de consumo relativamente arraigados, además cuenta con una dilatada experiencia en la administración de su poder adquisitivo. Ha pasado distintas etapas de su vida en las que ha tenido que adecuar su consumo a las circunstancias individuales (ingresos), familiares (ciclos de vida) e históricas (privaciones). Pero por otra parte sus grandes 
necesidades suelen estar cubiertas, aunque surgen otras nuevas, las cuales van a generar nuevas demandas, hasta ahora poco atendidas por el mercado.

Con la jubilación también se inicia el proceso llamado de despatrimonialización (INSERSO, 1990). La transmisión de la herencia ante mortem es una realidad en uno de cada cinco casos, en contra de lo que recomiendan notarios y abogados. Diferentes estudios (Goddy, 1993; Grundy,1983), hacen notar que el reparto y cesión de la herencia a los herederos, antes del fallecimiento, repercute negativamente en el propio bienestar del anciano. Le despoja de una fuente importante de sanción a sus posibles herederos, aunque con ello se logre apaciguar las tensiones intergeneracionales. Por el contrario, la herencia post mortem le garantiza su cuidado personal y afectivo más que cualquier otro hecho. Muchos mayores preferirían no decidir el reparto de la herencia hasta el final de su vida, en función de cómo sea el trato recibido por sus hijos, familiares, estando este asunto relacionado con el reparto de la carga en el cuidado y con el lugar de residencia del mayor. Por ello, la mayoría de los españoles parece haber optado por guardar la carta de herencia, y no jugarla en vida, aunque hay una serie de variables que influyen sobre este fenómeno: el hecho de quedar viudo/a anima a solucionar los papeles de la herencia, mientras que el estar casado ralentiza el proceso, el tipo de hábitat (en el medio rural se da una mayor disposición a dejar resuelta la herencia), la clase social, el estado de salud, la ocupación anterior, son también variables que afectan al proceso, sin reflejar pautas demasiado ordenadas.

Como consecuencia de la dificultad que tienen en nuestra sociedad los jóvenes para poder independizarse, para algunos expertos (Iglesias de Ussel,1988: Rivas y Vara,1989), se produce un cierto adelanto de la herencia que en circunstancias normales este trasvase de patrimonio a los hijos sólo tendría lugar tras el fallecimiento de uno de los cónyuges. Incluso se ha constatado la presencia de "herencias negativas", al apoyar los padres a los hijos donde la descapitalización que 
hubieron de soportar para adelantar la transmisión del patrimonio, puede dejar a los padres a merced de la solidaridad filial.

\subsection{Las ayudas económicas familiares}

Las relaciones familiares, sobre todo de padres e hijos, que ocurren en la vejez, están formadas por un complejo entramado de intercambios tanto económicos como afectivos, así lo demuestra una encuesta del INSERSO de 1.990. Sus resultados reflejan como el 15\% de los españoles de 65 y más años reciben ayudas económicas de sus familiares, de forma habitual u ocasional. Pero los flujos de dinero más frecuentes se producen en sentido inverso, de padres a hijos. Un 35\% de las personas mayores entrevistadas declara dar, ocasional o habitualmente, este tipo de ayuda monetaria a sus familiares (INSERSO, 90).

Según los resultados de la tabla una quinta parte $(19 \%)$ responde que no recibe de sus hijos o de algún familiar un dinero regular, "aunque no le importaría recibirlo". El rotundo "no, y tampoco lo aceptaría" lo contesta uno de cada tres mayores (35\%). El resto $(38 \%)$ responde negativamente, pero sin decantarse en ninguna de las opciones anteriores. Las respuestas extremas, se deben a que las mujeres de superior edad son las que más confiesan recibir dinero, y los varones jóvenes los que menos. Estos, en cambio, sobresalen por el rechazo a este tipo de ayudas familiares.

Tabla 12. Ayudas monetaria

\begin{tabular}{|c|c|c|c|c|c|}
\hline & \multirow[b]{2}{*}{ Edad } & \multicolumn{4}{|c|}{ Recibe dinero regularmente } \\
\hline & & Sí & $\begin{array}{l}\text { No, y no le } \\
\text { importaría }\end{array}$ & No, y no lo aceptaría & \begin{tabular}{|l} 
No, \\
otras \\
razones
\end{tabular} \\
\hline \multirow{2}{*}{ Varones } & -75 & 3 & 20 & 41 & 36 \\
\hline & $75+$ & 5 & 17 & 34 & 43 \\
\hline \multirow{2}{*}{ Mujeres } & -75 & 10 & 19 & 35 & 37 \\
\hline & $75+$ & 12 & 19 & 31 & 38 \\
\hline
\end{tabular}




\begin{tabular}{|l|l||l|l||l|l||}
\hline \multirow{2}{*}{ Total } & Varones & 4 & 19 & 39 & 38 \\
\cline { 2 - 6 } & Mujeres & 11 & 19 & 32 & 38 \\
\hline \hline \multirow{2}{*}{ Total } & -75 & 7 & 20 & 37 & 36 \\
\hline Total & 9 & 18 & 32 & 40 \\
\hline \hline
\end{tabular}

El dinero procedente de los hijos (generalmente), que reciben tan sólo ocho de cada diez ancianos, no suele quedarse sin contrapartida. Casi la mitad de los que reciben esta ayuda monetaria (43\%), dice cuidar frecuentemente a los nietos, y más de la mitad (57\%) ayuda a los hijos en otras tareas. Por otro lado, se demuestra que estas contrapartidas en especie forman parte de una clase de relaciones de economía y afecto en las que suele primar este último. El ejemplo modelo está en los que no aceptarían dinero de los hijos y, sin embargo, se encuentran a la cabeza en el cuidado de los nietos y otras ayudas.

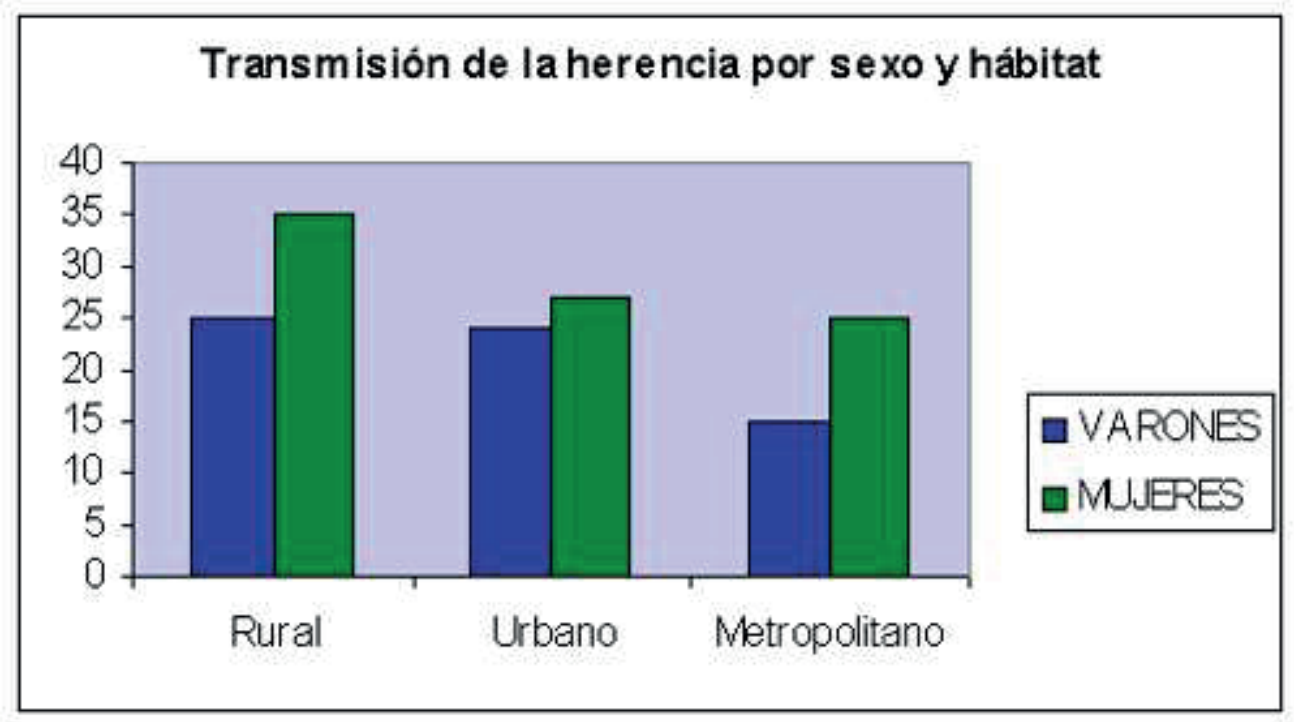

Tabla 13. Ayudas Familiares

\begin{tabular}{|l|c|c|}
\hline \multicolumn{1}{|c||}{} & \multicolumn{2}{c|}{ Realizan con frecuencia actividades de... } \\
\cline { 2 - 3 } & Cuidar nietos & Ayudar hijos \\
\hline Recibe dinero de hijos & \\
\hline
\end{tabular}




\begin{tabular}{|l||l|l|}
\hline Si & 43 & 57 \\
\hline No, no le impotaría & 46 & 45 \\
\hline No, y no lo aceptaría & 54 & 48 \\
\hline No, otras situaciones & 38 & 37 \\
\hline Total & $\mathbf{4 6}$ & $\mathbf{4 4}$ \\
\hline
\end{tabular}

La familia juega un papel de suma importancia en la calidad de vida de nuestros mayores, en cuanto fuente de auxilio, compañía y afecto; de manera especial cuando el anciano se convierte en más "dependiente" de la cooperación de otros. Esto, se hace notable en los casos en los que carece de cónyuge o cuando ambos cónyuges se hallan en mal estado de salud físico o psíquico, no pudiendo, en consecuencia, ayudarse mutuamente. Es entonces cuando más se precisa de la colaboración de los hijos, si los hubiese, o de otros familiares. Hasta llegar a una situación extrema, coincidente con la última etapa de la vida, muchos padres no han dejado de atender a sus hijos (tanto económica como asistencialmente), aunque estos ya se encuentren emancipados y hayan formado un núcleo familiar propio. Hasta tal punto esto es así que, según la información proporcionada por el INSERSO (1990), aproximadamente la mitad de los niños menores de cinco años quedan regularmente al cuidado de sus abuelas cuando sus padres, por razones laborales o de ocio, no pueden hacerlo. La mayor parte de las veces, los abuelos no viven en la misma casa de sus nietos. Pero esto no se convierte en un obstáculo para prestar este servicio familiar. 


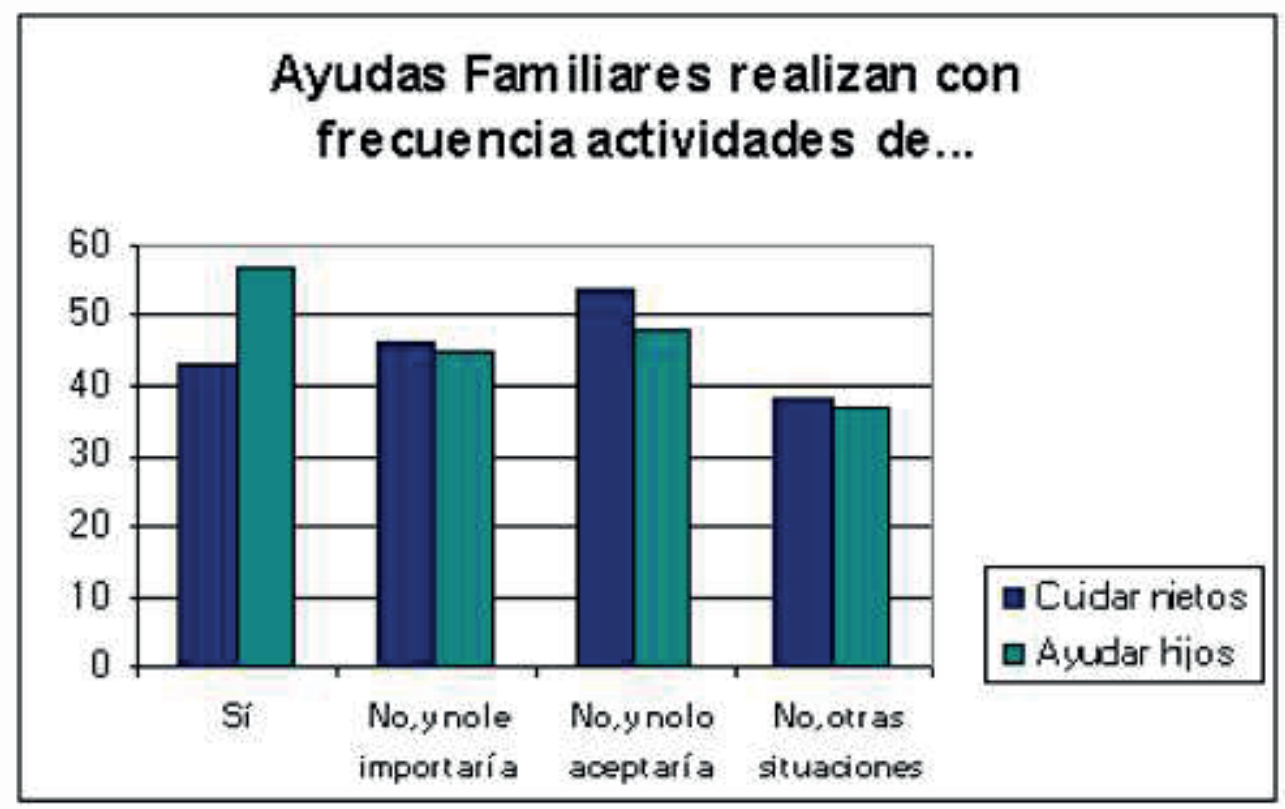

Otro elemento de carácter económico es el papel de las pensiones en las economías domésticas; esto ha sido señalado por diferentes investigadores (Pérez Ortiz, 1988; Bazo, 1996)las pensiones de los mayores en España tienen una importante función económica complementaria para las familias. En más de un tercio de los hogares españoles entra una pensión de la Seguridad Social, lo que representa casi 7 billones de pesetas al año, que desde los bancos y sobre todo desde las cajas circulan animando la economía española (Gaviria, 1996). También es importante la generosidad de los abuelos para con sus nietos, siendo más permisivos y generosos de lo que lo fueron con sus hijos. Los abuelos atienden a los caprichos de los nietos mediante regalos y aportando ayudas en metálico a los hijos para que compren ropa y juguetes a los más pequeños.

\section{Bibliografía}

Amando de Miguel (1994-1995), La sociedad española. Persona y sociedad en la vejez. 
Bazo, M.T. (1.998). "Aportaciones de las personas mayores a la sociedad". REIS N 73. CIS. Madrid.

CIS (1.993), "Apoyo informal a las personas mayores", Estudio no 2.072.

Fericgla, J.M. (1.992). "Envejecer. Una antropología de la ancianidad". Antthropos, 92:134. Barcelona.

Fernández Viguera, B. (1.995). "Género Social y procesos de empobrecimiento". En VVAA: Desigualdad y pobreza hoy". Talasa, Madrid.

Gaviria, M. (1.996). La séptima potencia. Ediciones B. Barcelona.

Gil Calvo, E. (1.993). Los recursos económicos de las personas mayores en España, Comunicación presentada en la Conferencia "Productos, bienes y sevicios para las personas mayores en el mercado con más futuro", Madrid, 23 y 24 de Noviembre.

Goddy, J. (1.993). The development of the family and marriage in Europe. Cambridge University Press.

Grande Estebán, I. (1.993). Marketing Estratégico para la Tercera Edad. ESIC, Madrid.

Grundy, J. (1983). "Demography and old age", Journal American Geriatric Society (vol. 31, 325-332).

Guillemard, A.M (1990). Analyse Comparée des Politiques de la Vieil-lesse en Europe, Madrid, Instituto Universitario de Sociología de Nuevas Técnologías, Universidad Autónoma de Madrid. 
Iglesias de Ussel, J. (1988)"La protección de la familia en España", en Papeles de Economía Española, N 77, FIES. CECA. Madrid.

INSERSO (1.990). "La tercera edad en España: necesidades y demandas". Madrid.

INSERSO (1.995). Las personas mayores en España. Perfiles. Reciprocidad. Ministerio de Asuntos Sociales. Madrid.

Pérez Ortiz, L. (1.998). Las necesidades de las personas mayores. INSERSO. Ministerio de Trabajo y Asuntos Sociales. Madrid.

Preston S. (1.984). Children and the Ederly: Divergents Paths for America's Dependents, Demography; 21 (4): 435-57.

Rivas, D. M, Vara, M. J. (1989). "Las unidades domésticas como amortiguamiento de la crisis económica", en VV.AA: Mujeres u hombres en la formación del pensamiento occidental. Universidad Autónoma de Madrid, vol. 1, pp. 447-153. Madrid.

Sánchez Vera P, (2.000). Los mayores y el consumo, en Medina Tornero M.E, Ruíz de Luna M.J (ed). Las políticas sociales para las personas mayores en el próximo siglo. $S$ P. Universidad de Murcia.

Toharia, L. (1.993). "Las desigualdades en el trabajo". Incluido en el I Simposio sobre Igualdad y Distribución de la Renta y la Riqueza, vol. IV (págs. 7-88). 